\title{
A note on strong-consistency of componentwise ARH(1) predictors
}

\author{
M. D. Ruiz-Medina †and J. Álvarez-Liébana \\ $\dagger$ Department of Statistics and Operation Research (mruiz@ugr.es, javialvaliebana@ugr.es) \\ Faculty of Sciences, University of Granada \\ Campus Fuente Nueva s/n \\ 18071 Granada, Spain
}

\begin{abstract}
This paper presents a new result on strong-consistency, in the trace norm, of a diagonal componentwise parameter estimator of the autocorrelation operator of an autoregressive process of order one (ARH(1) process), allowing strong-consistency of the associated plug-in predictor. These results are derived, when the eigenvectors of the autocovariance operator are unknown, and the autocorrelation operator does not admit a diagonal spectral representation with respect to the eigenvectors of the autocovariance operator.

Keywords: Dimension reduction techniques, empirical orthogonal bases, functional prediction, strong-consistency, trace norm

2010 Mathematics Subject Classification Primary 60G10; 60G15. Secondary 60F99; 60J05; 65F 15
\end{abstract}

\section{Introduction.}

It is well-known that functional prediction from the $\mathrm{ARH}(1)$ framework (see Bosq [6] and Bosq and Blanke [7]) has become a very active research area, driven by its interesting applications in the analysis of high-dimensional data (see, for example, Álvarez-Liébana, Bosq and Ruiz-Medina [1]). Several authors have studied the asymptotic properties of componentwise estimators of the autocorrelation operator, and $\mathrm{ARH}(1)$ predictors, in the case of known and unknown eigenvectors of the autocovariance operator. We refer to $[9,10,11,12]$, where the efficiency, consistency and asymptotic normality of 
these estimators are addressed, in a parametric framework (see also ÁlvarezLiébana, Bosq and Ruiz-Medina [2], on weak consistency in the norm of Hilbert-Schmidt operators). In this paper, we pay attention to the diagonal componentwise consistent estimation of the autocorrelation operator of an $\mathrm{ARH}(1)$ process, from projection into the empirical eigenvectors of the autocovariance operator, when their theoretical counterparts do not diagonalize the autocorrelation operator. A suitable sample-size-dependent truncation order, according to the spectral properties of the autocovariance operator of an $\mathrm{ARH}(1)$ process (namely, decay velocity and distance between eigenvalues), is usually selected to obtain the desirable asymptotic properties of componentwise parameter estimators, and their associated plug-in predictors, under different setting of conditions. The usual assumption on the autocorrelation operator is its boundedness or Hilbert-Schmidt property. Hence, the error term is usually measured in both operator norms, the norm of the space of bounded linear operators and the Hilbert-Schmidt norm. In the same way, dimension reduction has been a crucial topic in the nonparametric and semiparametric frameworks. In these contexts, a kernel-based predictor is usual adopted, as early formulated in [13]. Additional covariates were incorporated in the novel semiparametric kernel-based proposal by [4], in which an extension, to the functional time series framework, of partial linear models, was firstly developed. In both frameworks, regular autocorrelation operators usually arise. Thus, the error term could be measured in the trace norm, in terms of the diagonal values, with the subsequent dimension reduction. This is the proposal of the note carried out here, which could help the usual computational limitations arising in the implementation of kernel-based estimators (see also [5]). A fractal approach was proposed in [8] to solve the curse of dimensionality, by imposing a concentration assumption about the distribution of the stochastic process. For example, in the Gaussian case, under the trace class assumption on the autocorrelation operator, we can go beyond the concentration assumption on the autocorrelation kernel values, characterizing the distributional properties of the underlying stochastic process. A diagonal design of the kernel-based estimator can then be considered, ensuring the desirable asymptotic properties.

This paper intends to focus the attention of the reader on the smoothing and regularization techniques usually applied in the functional data context, that allow to work on a trace-class autocorrelation operator context, and hence, to measure the autocorrelation operator estimation error, in the trace norm. Particularly, strong-consistency in the trace norm also implies the 
consistency in the norm of the space of Hilbert-Schmidt operators, as well as in the norm of bounded linear operators. Results derived in this paper are mainly motivated by this fact, allowing an important dimension reduction, in the componentwise estimation of the autocorrelation operator of an $\mathrm{ARH}(1)$ process, and its plug-in prediction. It is important to highlight that the distributional properties of a wide class of stochastic processes in time and/or space can be characterized in terms of stochastic evolution equations, involving trace and positive autocorrelation operators (see, for example, Anh, Leonenko and Ruiz-Medina [3], in the spatiotemporal case).

\section{Preliminaries.}

Let $H$ be a real separable Hilbert space, and let $X=\left\{X_{n}, n \in \mathbb{Z}\right\}$ be a zero-mean $\mathrm{ARH}(1)$ process on the basic probability space $(\Omega, \mathcal{A}, P)$ satisfying the following equation:

$$
X_{n}=\rho\left(X_{n-1}\right)+\varepsilon_{n}, n \in \mathbb{Z},
$$

where $\rho$ is the autocorrelation operator of process $X$, which belongs to the space $\mathcal{L}(H)$ of bounded linear operators, such that $\left\|\rho^{k}\right\|_{\mathcal{L}(H)}<1$, for $k \geq k_{0}$, and for some $k_{0}$. Here, as usual, $\|\cdot\|_{\mathcal{L}(H)}$ denotes the norm in the space $\mathcal{L}(H)$. The Hilbert-valued innovation process $\varepsilon=\left\{\varepsilon_{n}, n \in \mathbb{Z}\right\}$ is assumed to be a strong white noise, and to be uncorrelated with the random initial condition. That is, $\varepsilon$ is a Hilbert-valued zero-mean stationary process, with independent and identically distributed components in time, and with $\sigma_{\varepsilon}^{2}=\mathrm{E}\left[\left\|\varepsilon_{n}\right\|_{H}^{2}\right]<\infty$, for all $n \in \mathbb{Z}$. We restrict our attention here to the case where $\rho$ is such that $\|\rho\|_{\mathcal{L}(H)}<1$.

Under the above-setting of conditions, involved in the introduction of equation (11), $X$ admits the following $\mathrm{MAH}(\infty)$ representation (see Bosq [6]):

$$
X_{n}=\sum_{k=0}^{\infty} \rho^{k}\left(\varepsilon_{n-k}\right), \quad n \in \mathbb{Z}
$$

that provides the unique stationary solution to equation (11). The autocovariance operator $C_{X}$ of the $\mathrm{ARH}(1)$ process $X$ is given by

$$
C_{X}=\mathrm{E}\left[X_{n} \otimes X_{n}\right]=\mathrm{E}\left[X_{0} \otimes X_{0}\right], \quad \forall n \in \mathbb{Z},
$$

which is assumed to be a trace operator. 
Let us now consider the empirical autocovariance operator $C_{n}$ of the ARH(1) process $X=\left\{X_{n}, n \in \mathbb{Z}\right\}$, computed as follows:

$$
C_{n}=\frac{1}{n} \sum_{i=0}^{n-1} X_{i} \otimes X_{i}, \quad n \geq 2,
$$

from a functional sample $X_{0}, X_{1}, \ldots, X_{n-1}$, of size $n$, of the ARH(1) process $X$. The empirical eigenvalues $\left\{C_{n, j}, j \geq 1\right\}$, and eigenvectors $\left\{\phi_{n, j}, j \geq 1\right\}$ of $C_{n}$ are given by (see [6], pp. 102-103):

$C_{n} \phi_{n, j}=C_{n, j} \phi_{n, j}, \quad j \geq 1, \quad C_{n, 1} \geq \cdots \geq C_{n, n} \geq 0=C_{n, n+1}=C_{n, n+2}=\ldots$

In the remainder, we will denote

$$
\begin{aligned}
X_{i, j, n} & =\left\langle X_{i}, \phi_{n, j}\right\rangle_{H}, \quad i \in \mathbb{Z}, \quad j \geq 1, n \geq 2, \\
\phi_{n, j}^{\prime} & =\operatorname{sgn}\left\langle\phi_{n, j}, \phi_{j}\right\rangle_{H} \phi_{j}, \quad j \geq 1, n \geq 2,
\end{aligned}
$$

where $\operatorname{sgn}\left\langle\phi_{n, j}, \phi_{j}\right\rangle_{H}=\mathbf{1}_{\left\langle\phi_{n, j}, \phi_{j}\right\rangle_{H} \geq 0}-\mathbf{1}_{\left\langle\phi_{n, j}, \phi_{j}\right\rangle_{H}<0}$. Thus,

$$
C_{n, j}=\frac{1}{n} \sum_{i=0}^{n-1} X_{i, j, n}^{2}, \quad j \geq 1, n \geq 2 .
$$

Let us now consider the cross-covariance operator $D_{X}$ given by

$$
D_{X}=\mathrm{E}\left[X_{i} \otimes X_{i+1}\right]=\mathrm{E}\left[X_{0} \otimes X_{1}\right], \quad \forall i \in \mathbb{Z},
$$

which is assumed to be a trace operator. The empirical cross-covariance operator $D_{n}$ is defined as follows:

$$
D_{n}=\frac{1}{n-1} \sum_{i=0}^{n-2} X_{i} \otimes X_{i+1}, \quad n \geq 2 .
$$

Given the empirical eigenvectors of $C_{n},\left\{\phi_{n, j}, j \geq 1\right\}$, we compute

$$
D_{n, j, l}^{*}=\left\langle D_{n}\left(\phi_{n, j}\right), \phi_{n, l}\right\rangle_{H}=\frac{1}{n-1} \sum_{i=0}^{n-2} X_{i, j, n} X_{i+1, l, n}, j, l \geq 1, n \geq 2,
$$

and, in particular, we will use the notation

$$
D_{n, j}=D_{n, j, j}^{*}=\left\langle D_{n}\left(\phi_{n, j}\right), \phi_{n, j}\right\rangle_{H}=\frac{1}{n-1} \sum_{i=0}^{n-2} X_{i, j, n} X_{i+1, j, n}, \quad j \geq 1, n \geq 2,
$$

where the empirical projections $\left\{X_{i, j, n}, j \geq 1, i=0,1, \ldots, n-1\right\}$ have been previously defined in equation (5). 


\section{Auxiliary results}

The auxiliary results presented in this section allow the derivation of Theorem 2 in the next section.

Assumption A1. The random initial condition $X_{0}$ of the $\mathrm{ARH}(1)$ process defined in (1) satisfies

$$
\mathrm{E}\left\{\left\|X_{0}\right\|_{H}^{4}\right\}<\infty
$$

Theorem 1. (see Theorem 4.1 on pp. 98-99, Corollary 4.1 on pp. 100-101 and Theorem 4.8 on pp. 116-117, in [6]). Under Assumption A1, for any $\beta>\frac{1}{2}$, as $n \rightarrow \infty$,

$$
\frac{n^{1 / 4}}{(\ln (n))^{\beta}}\left\|C_{n}-C_{X}\right\|_{\mathcal{S}(H)} \rightarrow^{a . s .} 0, \quad \frac{n^{1 / 4}}{(\ln (n))^{\beta}}\left\|D_{n}-D_{X}\right\|_{\mathcal{S}(H)} \rightarrow^{a . s .} 0
$$

and, if $\left\|X_{0}\right\|_{H}$ is bounded,

$$
\begin{gathered}
\left\|C_{n}-C_{X}\right\|_{\mathcal{S}(H)}=\mathcal{O}\left(\left(\frac{\ln (n)}{n}\right)^{1 / 2}\right) \text { a.s., } \\
\left\|D_{n}-D_{X}\right\|_{\mathcal{S}(H)}=\mathcal{O}\left(\left(\frac{\ln (n)}{n}\right)^{1 / 2}\right) \text { a.s. }
\end{gathered}
$$

where $\|\cdot\|_{\mathcal{S}(H)}$ denotes the norm of the Hilbert-Schmidt operators on H, and a.s. means, as usual, almost surely.

In the following, we denote by $\left\{C_{j}, j \geq 1\right\}$ the sequence of eigenvalues of the autocovariance operator $C_{X}$, satisfying

$$
C_{X}\left(\phi_{j}\right)=C_{j} \phi_{j}, \quad j \geq 1,
$$

with $\left\{\phi_{j}, j \geq 1\right\}$ being the associated system of eigenvectors.

Lemma 1. Under Assumption A1, for $n$ sufficiently large,

$$
\frac{n^{1 / 4}}{(\ln (n))^{\beta}} \sup _{j \geq 1}\left|C_{n, j}-C_{j}\right| \leq \frac{n^{1 / 4}}{(\ln (n))^{\beta}}\left\|C_{n}-C\right\|_{\mathcal{S}(H)} \rightarrow^{a . s .} 0,
$$

where $\left\{C_{j}, j \geq 1\right\}$ have been introduced in equation (11). 
The proof of Lemma 1 is straightforward since, from Theorem 1 and $n$ sufficiently large, $C_{n}$ is a Hilbert-Schmidt operator, and in particular, it is a compact operator. Thus, applying Lemma 4.2 on p. 103 in [6], and Theorem 1, for $n \geq n_{0}$, with $n_{0}$ sufficiently large, we obtain

$$
\frac{n^{1 / 4}}{(\ln (n))^{\beta}} \sup _{k \geq 1}\left|C_{n, k}-C_{k}\right| \leq \frac{n^{1 / 4}}{(\ln (n))^{\beta}}\left\|C_{n}-C_{X}\right\|_{\mathcal{S}(H)} \rightarrow^{a . s .} 0 .
$$

Proposition 1 below obtains the strong-consistency of $\left\{D_{n, j}, j \geq 1\right\}$, introduced in equation (8). In the derivation of these strong-consistency results, the distance between the eigenvalues of the autocovariance operator, and their rate of convergence to zero, will play a key role. Namely, if the following quantities are considered:

$$
\Lambda_{k}=\sup _{1 \leq j \leq k}\left(C_{j}-C_{j+1}\right)^{-1}, \quad k \geq 1,
$$

the rate of divergence of $\Lambda_{k_{n}}$ is crucial, as can be noted in the statements of Lemma 2 and Proposition 1 (see also Remark 1 below). Henceforth, $k_{n}$ denotes a truncation parameter, which verifies

$$
\lim _{n \rightarrow \infty} k_{n}=\infty, \quad \frac{k_{n}}{n}<1, \quad k_{n} \geq 1 .
$$

Lemma 2. Let us consider $\Lambda_{k_{n}}$ introduced in equation (14), for a given truncation parameter $k_{n}$, as reflected in (15). Assuming that $\left\|X_{0}\right\|_{H}$ is bounded and $\Lambda_{k_{n}}=o\left(n^{1 / 4}(\ln (n))^{\beta-1 / 2}\right)$, as $n \rightarrow \infty$, under Assumption A1, the following limit then holds

$$
\frac{n^{1 / 4}}{(\ln (n))^{\beta}} \sup _{1 \leq j \leq k_{n}}\left\|\phi_{n, j}^{\prime}-\phi_{n, j}\right\|_{H} \rightarrow^{a . s .} 0, \quad n \rightarrow \infty,
$$

for any $\beta>1 / 2$, where $\left\{\phi_{n, j}^{\prime}, j \geq 1\right\}$ are introduced in equation (5).

Proof. From equation (4.44) in Lemma 4.3 on page 104 of [6], for any $n \geq 2$ and $1 \leq j \leq k_{n}$,

$$
\left\|\phi_{n, j}^{\prime}-\phi_{n, j}\right\|_{H} \leq a_{j}\left\|C_{n}-C\right\|_{\mathcal{L}(H)} \leq 2 \sqrt{2} \Lambda_{k_{n}}\left\|C_{n}-C\right\|_{\mathcal{S}(H)},
$$

which implies that

$$
\mathcal{P}\left(\sup _{1 \leq j \leq k_{n}}\left\|\phi_{n, j}^{\prime}-\phi_{n, j}\right\|_{H} \geq \eta\right) \leq \mathcal{P}\left(\left\|C_{n}-C\right\|_{\mathcal{S}(H)} \geq \frac{\eta}{2 \sqrt{2} \Lambda_{k_{n}}}\right)
$$


Thus, since $\left\|X_{0}\right\|_{H}$ is bounded, from Theorem 4.2 on pp. 99-100 of [6], under Assumption A1 for any $\eta>0$, and $\beta>1 / 2$,

$$
\begin{aligned}
& \mathcal{P}\left(\frac{n^{1 / 4}}{(\ln (n))^{\beta}} \sup _{1 \leq j \leq k_{n}}\left\|\phi_{n, j}^{\prime}-\phi_{n, j}\right\|_{H} \geq \eta\right) \\
\leq & \mathcal{P}\left(\left\|C_{n}-C\right\|_{\mathcal{S}(H)} \geq \frac{\eta}{2 \sqrt{2} \Lambda_{k_{n}}} \frac{(\ln (n))^{\beta}}{n^{1 / 4}}\right) \\
\leq & 4 \exp \left(-\frac{n \frac{\eta^{2}}{8 \Lambda_{k_{n}}^{2}} \frac{(\ln (n))^{2 \beta}}{n^{1 / 2}}}{\gamma_{1}+\delta_{1} \frac{\eta}{2 \sqrt{2} \Lambda_{k_{n}}} \frac{(\ln (n))^{\beta}}{n^{1 / 4}}}\right) \\
= & \mathcal{O}\left(n-\frac{\eta^{2}}{\gamma_{1}+\eta \delta_{1}\left(\frac{\ln (n)}{n}\right)^{1 / 2}}\right), \quad n \rightarrow \infty .
\end{aligned}
$$

Thus, taking $\eta^{2}>\gamma_{1}+\delta_{1} \eta$, sequence (19) is summable, and applying BorelCantelli Lemma we arrive to the desired result.

Proposition 1. Under the conditions of Lemma 圆, considering Assumption A1, for $\beta>\frac{1}{2}$, and $n$ sufficiently large,

$$
\frac{n^{1 / 4}}{(\ln (n))^{\beta}} \sup _{j \geq 1}\left|D_{n, j}-D_{j}\right| \rightarrow^{\text {a.s. }} 0, \quad n \rightarrow \infty,
$$

where $\left\{D_{n, j}, j \geq 1\right\}$ are defined in equation (8), and $\left\{D_{j}, j \geq 1\right\}$ are given by $D_{j}=D_{X}\left(\phi_{j}\right)\left(\phi_{j}\right)$, for every $j \geq 1$, with, as before, $\left\{\phi_{j}, j \geq 1\right\}$ being the system of eigenvectors of $C_{X}$.

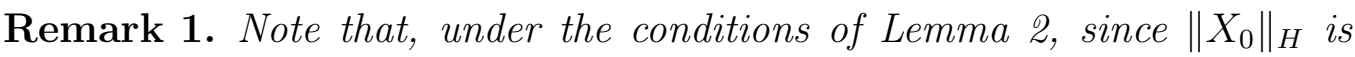
bounded, from Theorem 1, the rate of convergence to zero in Lemma 1 and Proposition 1 can be improved up to the value $\frac{(\ln (n))^{\beta}}{n^{\gamma}}$, for $\beta>1 / 2$, and $\gamma \in(1 / 4,1 / 2)$, allowing larger values of the truncation order $k_{n}$, given by (15), for a fixed sample size $n$.

Proof. From Theorem 1, there exists an $n_{0}$ such that for $n \geq n_{0}, D_{n}$ is a 
Hilbert-Schmidt operator. Then, for $n \geq n_{0}$, and for every $j$,

$$
\begin{aligned}
& \frac{n^{1 / 4}}{(\ln (n))^{\beta}}\left|D_{n, j}-D_{j}\right|=\frac{n^{1 / 4}}{(\ln (n))^{\beta}} \\
& \quad \times \mid D_{n}\left(\phi_{n, j}\right)\left(\phi_{n, j}\right)-D_{n}\left(\phi_{n, j}\right)\left(\phi_{j}\right)+D_{n}\left(\phi_{n, j}\right)\left(\phi_{j}\right) \\
& \quad-D\left(\phi_{n, j}\right)\left(\phi_{j}\right)+D\left(\phi_{n, j}\right)\left(\phi_{j}\right)-D\left(\phi_{j}\right)\left(\phi_{j}\right) \mid \\
& \leq \frac{n^{1 / 4}}{(\ln (n))^{\beta}}\left[\left\|D_{n}\left(\phi_{n, j}\right)\right\|_{H}\left\|\phi_{n, j}-\phi_{j}\right\|_{H}+\left\|\left(D_{n}-D\right)\left(\phi_{n, j}\right)\right\|_{H}\left\|_{\phi_{j}}\right\|_{H}\right. \\
& \left.\quad+\left\|D\left(\phi_{n, j}-\phi_{j}\right)\right\|_{H}\left\|\phi_{j}\right\|_{H}\right] \\
& \leq \frac{n^{1 / 4}}{(\ln (n))^{\beta}}\left[\left\|D_{n}\right\|_{\mathcal{L}(H)}\left\|\phi_{n, j}-\phi_{j}\right\|_{H}+\left\|D_{n}-D\right\|_{\mathcal{L}(H)}\right. \\
& \left.\quad+\|D\| \mathcal{L}(H)\left\|\phi_{n, j}-\phi_{j}\right\|_{H}\right],
\end{aligned}
$$

where, as before, $\left\{\phi_{j}, j \geq 1\right\}$ and $\left\{\phi_{n, j}, j \geq 1\right\}$ respectively denote the theoretical and empirical eigenvectors of $C_{X}$, and $\|\cdot\|_{\mathcal{L}(H)}$ denotes the norm in the space of bounded linear operators.

From Theorem 1 ,

$$
\frac{n^{1 / 4}}{(\ln (n))^{\beta}}\left\|D_{n}-D\right\|_{\mathcal{L}(H)} \leq \frac{n^{1 / 4}}{(\ln (n))^{\beta}}\left\|D_{n}-D\right\|_{\mathcal{S}(H)} \rightarrow^{a . s .} 0,
$$

and, for $n$ sufficiently large, $\left\|D_{n}\right\|_{\mathcal{L}(H)}<\infty$. Furthermore, from Lemma 2 (see equation (16)),

$$
\frac{n^{1 / 4}}{(\ln (n))^{\beta}} \sup _{1 \leq j \leq k_{n}}\left\|\phi_{n, j}-\phi_{j}\right\|_{H} \rightarrow^{a . s .} 0 .
$$

Hence, from equations (22) and (23), taking the supremum in $j$ at the lefthand side of equation (21), we obtain equation (20).

\section{Strong-consistency in the trace operator norm}

In the subsequent developments, we assume that the eigenvectors $\left\{\phi_{j}, j \geq 1\right\}$ of $C_{X}$ are unknown. The diagonal componentwise estimator of $\rho$ formulated below is then defined in terms of the empirical eigenvectors.

The following condition is assumed in the remainder of this section:

Assumption A2. The empirical eigenvalue $C_{n, k_{n}}>0$ a.s, where $k_{n}$ is the truncation parameter satisfying the conditions established in (15). 
Under Assumption A2, from a functional sample of size $n, X_{0}, \ldots, X_{n-1}$, the following estimator $\widehat{\rho}_{k_{n}}$ of $\rho$ is formulated:

$$
\widehat{\rho}_{k_{n}}=\sum_{j=1}^{k_{n}} \rho_{n, j} \phi_{n, j} \otimes \phi_{n, j}=\sum_{j=1}^{k_{n}} \frac{D_{n, j}}{C_{n, j}} \phi_{n, j} \otimes \phi_{n, j},
$$

where, for each $j \geq 1$ and $n \geq 2$,

$$
\rho_{n, j}=\frac{D_{n, j}}{C_{n, j}}=\frac{\frac{1}{n-1} \sum_{i=0}^{n-2}\left\langle X_{i}, \phi_{n, j}\right\rangle_{H}\left\langle X_{i+1}, \phi_{n, j}\right\rangle_{H}}{\frac{1}{n} \sum_{i=0}^{n-1}\left[\left\langle X_{i}, \phi_{n, j}\right\rangle_{H}\right]^{2}}=\frac{n}{n-1} \frac{\sum_{i=0}^{n-2} X_{i, j, n} X_{i+1, j, n}}{\sum_{i=0}^{n-1} X_{i, j, n}^{2}} .
$$

The strong-consistency of $\widehat{\rho}_{k_{n}}$, in the trace norm, is derived in the following result under suitable conditions.

Theorem 2. Under Assumption A2, and the conditions assumed in Lemma 2, if $\rho$ is a positive trace operator, then, $\widehat{\rho}_{k_{n}}$, introduced in equations (24)(25), is strongly-consistent in the trace norm, i.e.,

$$
\left\|\rho-\widehat{\rho}_{k_{n}}\right\|_{1} \rightarrow^{a . s .} 0, \quad n \rightarrow \infty
$$

for $k_{n}=o\left(\frac{n^{1 / 4}}{(\ln (n))^{\beta}}\right)$, as $n \rightarrow \infty$. Here, $\|\cdot\|_{1}$ denotes the trace operator norm. Consequently, $\widehat{\rho}_{k_{n}}$ is also strongly-consistent in the spaces $\mathcal{S}(H)$ and $\mathcal{L}(H)$.

Proof. From Theorem 1, we have, for $n$ sufficiently large,

$$
\begin{aligned}
& \left\|D_{n} C_{n}^{-1}-D_{X} C_{X}^{-1}\right\|_{\mathcal{S}(H)} \\
& =\left\|D_{n} C_{n}^{-1}-D_{X} C_{n}^{-1}+D_{X} C_{n}^{-1}-D_{X} C_{X}^{-1}\right\|_{\mathcal{S}(H)} \\
& \leq\left\|D_{n} C_{n}^{-1}-D_{X} C_{n}^{-1}\right\|_{\mathcal{S}(H)}+\left\|D_{X} C_{n}^{-1}-D_{X} C_{X}^{-1}\right\|_{\mathcal{S}(H)} \\
& =\left\|\left(D_{n}-D_{X}\right) C_{n}^{-1}\right\|_{\mathcal{S}(H)}+\left\|D_{X}\left(C_{n}^{-1}-C_{X}^{-1}\right)\right\|_{\mathcal{S}(H)},
\end{aligned}
$$

since from such a theorem, $D_{X} C_{n}^{-1} \in \mathcal{S}(H)$ for $n$ sufficiently large. Again, from Theorem 1, equation (27) tends to zero as $n \rightarrow \infty$, a.s., which means that $\left\|D_{n} C_{n}^{-1}-D_{X} C_{X}^{-1}\right\|_{\mathcal{S}(H)}$ also converges to zero a.s. From Theorem 1 and equation (27), we also have that there exists an $n_{0} \in \mathbb{N}$ such that, for $n \geq n_{0}$, 
$D_{n} C_{n}^{-1}$ is a positive trace operator almost surely. Considering now $n \geq n_{0}$, for any orthonormal basis $\left\{\varphi_{j}, j \geq 1\right\}$ on $H$,

$$
\left\|D_{n} C_{n}^{-1}\right\|_{1}=\sum_{j=1}^{\infty}\left\langle D_{n} C_{n}^{-1}\left(\varphi_{j}\right), \varphi_{j}\right\rangle_{H}
$$

Note also that

$$
\left\|D_{X} C_{X}^{-1}\right\|_{1}=\sum_{j=1}^{\infty}\left\langle D_{X} C_{X}^{-1}\left(\varphi_{j}\right), \varphi_{j}\right\rangle_{H} .
$$

Furthermore, from Lemma 1, as $n \rightarrow \infty, C_{n, j}^{-1}$ converges a.s. to $C_{j}^{-1}$, uniformly in $j$, with convergence rate at least $\frac{(\ln (n))^{\beta}}{n^{1 / 4}}$, for $\beta>1 / 2$. The same assertion holds for the uniform a.s. convergence of $D_{n, j}$ to $D_{j}$, as $n \rightarrow \infty$, from Proposition 1. Consequently, for every $j$, as $n \rightarrow \infty, D_{n, j} C_{n, j}^{-1}$ converges a.s. to $D_{j} C_{j}^{-1}$, with uniform a.s. rate of convergence at least $\frac{(\ln (n))^{\beta}}{n^{1 / 4}}$, for $\beta>1 / 2$. Equivalently, from equation (12) in Lemma 1, and equation (20) in Proposition 1,

$$
\begin{aligned}
\left\|\widehat{\rho}_{k_{n}}\right\|_{1}= & \sum_{j=1}^{k_{n}} D_{n, j} C_{n, j}^{-1} \\
& \leq f(n)=\mathcal{O}\left(k_{n} \frac{(\ln (n))^{\beta}}{n^{1 / 4}}+\sum_{j=1}^{\infty} D_{j} C_{j}^{-1}\right), n \rightarrow \infty \text { a.s. }
\end{aligned}
$$

Since $k_{n}=o\left(\frac{n^{1 / 4}}{(\ln (n))^{\beta}}\right)$, equation (29) converges a.s., when $n \rightarrow \infty$, to

$$
\left\|D_{X} C_{X}^{-1}\right\|_{1}=\sum_{j=1}^{\infty} D_{j} C_{j}^{-1}=\sum_{j=1}^{\infty} D_{X}\left(\phi_{j}\right)\left(\phi_{j}\right)\left[C_{X}\left(\phi_{j}\right)\left(\phi_{j}\right)\right]^{-1}=\|\rho\|_{1} .
$$

For two positive trace operators, $\mathcal{K}$ and $\mathcal{T}$, the following identities are satisfied, for any orthonormal basis $\left\{\varphi_{j}, j \geq 1\right\}$ on $H$,

$$
\begin{aligned}
\|\mathcal{K}-\mathcal{T}\|_{1} & =\operatorname{trace}(|\mathcal{K}-\mathcal{T}|)=\max \left(\|\mathcal{K}\|_{1},\|\mathcal{T}\|_{1}\right)-\min \left(\|\mathcal{K}\|_{1},\|\mathcal{T}\|_{1}\right) \\
\|\mathcal{K}\|_{1} & =\sum_{j=1}^{\infty}\left\langle\mathcal{K}\left(\varphi_{j}\right), \varphi_{j}\right\rangle_{H}, \quad\|\mathcal{T}\|_{1}=\sum_{j=1}^{\infty}\left\langle\mathcal{T}\left(\varphi_{j}\right), \varphi_{j}\right\rangle_{H} .
\end{aligned}
$$


In particular, for $n \geq n_{0}$,

$$
\left\|\rho-\widehat{\rho}_{k_{n}}\right\|_{1}=\max \left(\|\rho\|_{1},\left\|\widehat{\rho}_{k_{n}}\right\|_{1}\right)-\min \left(\|\rho\|_{1},\left\|\widehat{\rho}_{k_{n}}\right\|_{1}\right) .
$$

The limit (26) then follows from equations (29) $-(31)$.

The strong consistency in $H$ of the associated ARH(1) plug-in predictor $\widehat{\rho}_{k_{n}}\left(X_{n-1}\right)$ of $X_{n}$ is now derived.

Corollary 1. Under the conditions of Theorem 2

$$
\left\|\widehat{\rho}_{k_{n}}\left(X_{n-1}\right)-\rho\left(X_{n-1}\right)\right\|_{H} \rightarrow^{a . s .} 0, \quad n \rightarrow \infty .
$$

Proof. The proof directly follows from Theorem 2, keeping in mind that the convergence in the trace norm implies the convergence in the space $\mathcal{L}(H)$ of bounded linear operators. Moreover,

$$
\left\|\widehat{\rho}_{k_{n}}\left(X_{n-1}\right)-\rho\left(X_{n-1}\right)\right\|_{H} \leq\left\|\widehat{\rho}_{k_{n}}-\rho\right\|_{\mathcal{L}(H)}\left\|X_{n-1}\right\|_{H} \rightarrow^{a . s .} 0, n \rightarrow \infty,
$$

since $\left\|X_{n-1}\right\|_{H}<\infty$.

Remark 2. Note that, when $\rho$ is Hilbert-Schmidt, but it is not positive trace operator, under the conditions of Lemma Q2, and if Assumption A2 holds, the following a.s. inequality is satisfied, as $n \rightarrow \infty$,

$$
\left\|\widehat{\rho}_{k_{n}}-\rho\right\|_{\mathcal{S}(H)}^{2} \leq\|\rho\|_{\mathcal{S}(H)}^{2}-\sum_{j=1}^{\infty}\left[\rho\left(\phi_{j}\right)\left(\phi_{j}\right)\right]^{2}=\sum_{j \neq k}^{\infty}\left[\frac{D_{X}\left(\phi_{j}\right)\left(\phi_{k}\right)}{C_{j}}\right]^{2}<\infty
$$

\section{Acknowledgments}

This work has been supported in part by project MTM2015-71839-P (co-funded by Feder funds), of the DGI, MINECO, Spain.

\section{References}

\section{References}

[1] J. Álvarez-Liébana, D. Bosq and M. D. Ruiz-Medina, Consistency of the plug-infunctional predictor of the OrnsteinUhlenbeck process in Hilbert and Banach spaces, Statistics and Probability Letters 117, 2016, 12-22. 
[2] J. Álvarez-Liébana, D. Bosq and M. D. Ruiz-Medina, Asymptotic properties of a componentwise $\mathrm{ARH}(1)$ plug-in predictor, J. Mult. Anal. 155, 2017, 12-34.

[3] V. V. Anh, N. N. Leonenko and M. D. Ruiz-Medina, Fractional-in-time and multifractional-in-space stochastic partial differential equations, Fractional Calculus and Applied Analysis 19, 2016, 1434-1459.

[4] G. Aneiros-Pérez and P. Vieu, Nonparametric time series prediction: a semi- functional partial linear modeling, J. Mult. Anal. 99, 2008, 834-857.

[5] A. Antoniadis, E. Paparoditis and T. Sapatinas, A functional waveletkernel approach for time series prediction, J. R. Stat. Soc. Ser. B. Stat. Methodol. 68, 2006, 837-857.

[6] D. Bosq, Linear Processes in Function Spaces, Springer, New York, 2000.

[7] D. Bosq and D. Blanke, Inference and Predictions in Large Dimensions, Wiley, Chichester, 2007.

[8] F. Ferraty, A. Goia and P. Vieu, Functional nonparametric model for time series: a fractal approach for dimension reduction, Test 11, 2002, 317-344.

[9] S. Guillas, Rates of convergence of autocorrelation estimates for autoregressive Hilbertian processes, Statistics $\& 3$ Probability Letters 55, 2001, 281-291.

[10] A. Mas, Normalité asymptotique de l'estimateur empirique de l'opérateur d'autocorrélation d'un processus $\mathrm{ARH}(1)$, C. R. Acad. Sci. Paris Sér. I Math. 329, 1999, 899-902.

[11] A. Mas, Consistance du prédicteur dans le modèle ARH(1): le cas compact, Ann. I.S.U.P. 48, 2004, 39-48.

[12] A. Mas, Weak-convergence in the functional autoregressive model, $J$. Multivariate Anal. 98, 2007, 1231-1261.

[13] J. M. Poggi, Prévision non paramétrique de la consommation électrique, Rev. Statistique Appliquée 42, 1994, 83-98. 